\title{
Application Characteristics of Fresh Traditional Chinese Medicine
}

\author{
Rui ZHANG ${ }^{1, a}$ and Ming-san MIAO ${ }^{2, b, *}$ \\ 1,2Hennan University of Chinese Medicine,Zhengzhou,China 450008 \\ azhangruiyaoli@126.com, ${ }^{\mathrm{b}}$ miaomingsan@163.com \\ ${ }^{*}$ Corresponding author
}

Keywords: Fresh medicine, Application, Characteristics, Curative effect.

\begin{abstract}
In the early years of Zhang Zhongjing, the characteristics and application of fresh herbs were described in detail. Fresh herbs without complex processing, convenient use, wide application, and high curative effect, fresh preserved products than medicinal active ingredient of medicine more, the efficacy is better than dry goods. The clinical application of fresh herbs also has some advantages, and the use of fresh herbs to treat many diseases has become one of the important methods of treatment of traditional Chinese medicine. This paper expounds the history and application characteristics of Chinese herbal medicine.
\end{abstract}

\section{Introduction}

Fresh herbal medicines are a kind of special Chinese herbal medicine including fresh botanicals and fresh animal medicine. The application of "fresh herbs" in China has not only a long history, but also a very rich resource. Through long clinical trials and experience accumulation, the successive physicians gradually formed a unique set of theoretical systems. "Fresh herbs" has become an important branch of the quintessence of Chinese medicine. With the rapid development of economy and the progress of medical and health care, the traditional methods of understanding and using fresh herbs have not fully satisfied the actual demand of medical treatment at present. The discipline of fresh herbs and its industrialization develop slowly; gradually widen the distance from modern science. Facing the new requirements and challenges, the fresh herbs must be carried out in the inheritance of breakthroughs and innovations.

\section{History of the Use of Fresh Chinese Medicine}

In the history of the development of fresh herbs, from the "Shen Nong tasted the hundred herbs, one day and encounter seventy poison", to put forward the "fresh use of Chinese medicine" and "living good" view. All the time, the curative effect of fresh herbs on preventing diseases has been paid much attention by doctors of ancient times. Fresh herbs are the first Chinese medicinal materials used to treat human diseases. The earliest use of the roots, bark, various hunting birds and animals, most of them are fresh goods, most are still used today, visible fresh drugs throughout the course of the origin and development of Chinese medicine. It is thus clear that the fresh herbal runs through the whole process of the origin and development of Chinese medicine. It is known that China most ancient Han traditional medicine prescription book "fifty-two preseriptions". It has taken "Take the two liters of stem yam, potato juice to take Gu impregnated, made of pulp, drink" [1]. This is used in the treatment of hemorrhoids (anal sores and bleeding edge). 
In Han Dynasty, Zhang Zhongjing paid special attention to the application of fresh herbs. His book "treatise on febrik and miscellaneous diseases" and "synopsis of golden chamber" with fresh medicine to treat various diseases has been described in detail.

In addition, Zhang Zhongjing's application of dried ginger, ginger and dried rehmannia root was continuously recognized by doctors of past dynasties. Zhang Zhongjing applies ginger to treat a variety of disorders. Such as: In Ramuli Cinnamomi Decoction, there is ginger help guizhi relieving musclesi and reconcile camp Wei. If vomiting, with ginger with bean soup to treat sweating too induced vomiting syndrome.

In the Tang Dynasty, thousand goldenprescription written by Sunsi Miao recorded with fresh reed rhizome, fresh bamboo shavings, rice, ginger, to treat nausea and retching without symptoms of food retching after typhoid fever

"Compendium of Materia Medica" in the Ming Dynasty there are more than 1100 fresh herbs with application. In the Ming Dynasty, Zhang Jingyue's "Yu Nü Jian formula" could be used to cure the coma caused by pathogenic heat. With the emergence of study of epidemic febrile disease, the development of fresh herbs reached its peak. It can be said that the clinical application of fresh herbs has been used by people since ancient times, and has certain practical value. In China's traditional Chinese medicine culture for thousands of years, fresh herbs always occupy a place, until the early liberation, the use of fresh herbs is still very common. But now the development of fresh medicine is facing many challenges. Fresh drug sources, storage and cultivation technology has a big problem, the majority of the dry goods instead of fresh goods [2]. In recent years the application of fresh traditional medicine shows a trend of shrinking. As a major characteristic of Chinese traditional treatment of diseases, we should strengthen the inheritance and development of fresh herbs.

\section{Application Characteristics of Fresh Traditional Chinese Medicine}

\section{Characteristics of Fresh Chinese Medicine}

China has rich resources of fresh herbs. Among the more than 2000 kinds of Chinese medicinal herbs, there are 486 kinds, which are mainly based on fresh use. The use of fresh herbs is one of the traditional characteristics of traditional Chinese medicine. Fresh herbs not only contain fresh, pure juice and smell, but also have pure natural properties. Fresh herbal medicinal can be directly applied to the clinic without complicated processing procedures. Compared with dried products, fresh herbs maintain the pure characteristics of natural medicines. Modern studies have shown that the active ingredients and pharmacological activities of fresh herbs are stronger than those of dry products.

Because of its fresh herbs are less than cold, warm dryness heats, cool to force more wins. The same kind of medicine, fresh herbs cold nature more than dry goods. The characteristics of cold fresh drug treatment on characteristics of febrile disease have a unique advantage [3]. According to the records of early febrile disease can be used fresh mint, kudzu, such as flowers, the middle can use fresh reed rhizome, Dendrobium, dandelion and other products. Later, we can use fresh yam, raw ground, milk and so on.

In addition, there are many fresh herbs have many features, including nourishing yin and cooling blood, slaking thirst, relieving summer-heat, resolving dampness and so on. It has a good curative effect on some hot symptoms, bleeding symptoms, acute 
disease, surgical diseases and difficult severe cases.

\section{Pharmacodynamic Characteristics of Fresh Traditional Chinese Medicine}

According to modern studies, many pharmacological activities of fresh herbs are very high. It in hypoglycemic, antioxidant, anti-inflammatory, anti-tumor and other aspects of treatment have played a very good effect. For example, fresh Houttuynia cordata has stronger effect on anti inflammation and bacteriostasis than dry products [4]. The effect of fresh motherwort capsule on the contraction of rat uterus in vitro is better than that of dried motherwort extract. Ginger decoction and decoction of dried ginger in the same dose, ginger decoction on rats induced by heat and antipyretic effects on rabbit eyelid conjunctiva stimulation reduction effect was significantly stronger than Ginger decoction [5]. The volatile oil content of fresh Perrin was significantly higher than that of dry products. At the same dose, the anti-inflammatory and amylase activities of fresh Perrin were higher than that of dry Perrin [6]. The contents of active substances, trace elements and amino acids in fresh animal medicine are obviously higher than those in dry products, and they have the effects of anticancer, righting and detoxifying. Experimental research shows that the medicine preparation with fresh gecko, fresh animal medicine formula of Agkistrodon acutus on murine cervical carcinoma (U14) tumor inhibitory rate and metastasis inhibition rates were significantly higher than the control group tumor model with high metastasis, metastasis of lung adenocarcinoma was significantly inhibited [7].

\section{Characteristics of the Usage of Fresh Chinese Medicine \\ Preservation Characteristics of Fresh Chinese Medicine}

Fresh herbs are a dynamic process in the process of picking. Color, taste, drug properties, and active ingredients change. If the preservation is improper, the medicinal material will also change color, take the oil, appear the enzyme solution, the reduction effect and so on [8]. Therefore, the preservation of fresh herbs is also an important aspect of the clinical application of fresh herbs [9]. Traditional Chinese medicine preservation methods are generally seasonal, easy to be affected by natural environmental factors, more restrictions on the use of conditions, the preservation of a short time and so on [10]. And the applicable medicinal materials have great limitations, mainly concentrated in the whole grass and small root medicine, the coverage is narrow. The traditional preservation measures cannot effectively solve mildew, moth, discoloration, and bleeding, weathering phenomenon. Therefore, although some methods are simple and convenient and low cost, they cannot achieve good utility when considering actual benefit [11]. Therefore, in the storage of fresh herbs, we should take reasonable and effective measures according to the nature of medicinal materials, pay attention to the application of modern scientific storage methods, and ensure the quality of fresh herbs.

\section{Characteristics of the Use of Fresh Chinese Medicine}

The usage method of fresh herbs has a long history. However, the traditional methods of using fresh medicine are only suitable for people's daily life. They are basically not adapted to the needs of modern clinical medication [12]. The research of Chinese fresh herbal medicine preparation is still in its infancy. At present, the fresh herbal medicine preparation in China is fresh motherwort capsule, Jinlong capsule, Jinshuixian capsule [13].

Compared with leonurus japonicus capsule, leonurus liquid extract and leonurus 
inspissant extract. Fresh motherwort capsule has the advantages of high component quantity, strong pharmacological effect and good clinical curative effect. And the preparation process is more advanced, less dosage, beautiful appearance, easy to carry. Jinlong Capsule is made from fresh bungarus parvus, fresh gecko, fresh fresh agkistrodon. Jinlong Capsule has significant inhibitory effect and anti recurrence and metastasis [14]. Jinlong Capsule can improve the clinical symptoms of tumor patients, reduce the cancer foci and improve the quality of life. Jinshuixian capsule is made from fresh bungarus parvus, fresh gecko, fresh American ginseng, cordyceps sinensis. It contains a variety of small molecular peptides, nucleotides, proteolytic enzymes and arginine, fatty acids, 19 free amino acids, 18 kinds of hydrolysis of amino acids and 19 kinds of beneficial trace elements, ginsenosides and so on [15]. This helps to improve the body's metabolism and energy metabolism, accelerate the repair of damaged tissues and promote the pathological cells to restore normal physiological functions.

\section{Conclusions}

The resources of Chinese medicinal herbs are very rich, and there are a large number of ancient books and rich experience in clinical use. So It should be fully developed and applied rationally. The Characteristic of fresh herbs is fresh and moist, rich in JinQi. It has been the people's favor. We treat diseases with fresh herbs, not only things good cheap; can solve the problem of lacking widely.

However, the development of fresh herbs still faces many problems. The main problems facing at present can be classified into two aspects: storage technology and the preparation of fresh medicinal preparations. Most of the fresh herbs used in clinic are drugs containing volatile, aromatic, protein and enzymes, peptides, vitamins and so on. These components will fail for a long time, and the heat decomposes easily. The development of our country's fresh herbs preparations is slow and the technical conditions are not complete. It is influenced by the traditional Chinese patent medicine formulation and technology.

The key to the development of fresh herbs is to solve the fresh-keeping technology. At present, the fresh-keeping technology of fresh medicine is simple but not durable, expensive but very troublesome. We should sum up the original preservation technology, find the shortcomings, and combine the existing advanced science and technology, so that fresh-keeping technology is cheap and practical. In addition, we should not simply pursue the preservation of the physical and chemical properties of fresh herbs, because they are still affected by many factors in the process of use, while the original efficacy of fresh herbs is impaired. We should strengthen the development of the fresh herbal preparations, excavate the effective prescriptions of Chinese ancient medical books and folk medicine, and apply them to the clinic effectively. We can make use of modern advanced pharmaceutical technology and production technology to study and produce new dosage forms at a high starting point.

\section{Acknowledgement}

Project supported by the Henan campus of Henan Province (CAI (2016) No. 38), Henan research and production project (172107000012), Zhengzhou research project (Zheng Keji 2016 (4)) 


\section{References}

[1] Jin Shiyuan.J. Capital Medicine, 2013, (01): 38-39.in Chinese.

[2] Peng Yong,Tan Fang. J. Capital Medicine, 2013, (05): 45-46.in Chinese.

[3] Liang Youqiang, Wang Kangzhan. J. GanSu Traditional Chinese Medicine, 2011, (02): 62-63.in Chinese.

[4] Lin Yunbin, Su Chaopin, Zhong Honglan, Shen Jian. J. Asia Pacific Traditional Medicine, 2014, (19): 4-5.in Chinese.

[5] Tao Jialei, Zhang Hongru. J. Li Shizhen Medicine and Materia Medica Research, 2014, (03): 689-690.in Chinese.

[6] Cao Guanhua, Xing Hanwen, Li Zedong, Zhao Ronghua, Chen Haifeng, Wang Bo, He Sen. J. Li Shizhen Medicine and Materia Medica Research, 2017, (02): 426-429.in Chinese.

[7] Ye Zaiqing.J.Modern Distance Education of Chinese Medicine. 2014 (16): 99-100+106.in Chinese.

[8] Wang Mengxi, Wu Qinan, Yue Wei, Liang Qiaoli, Gu Wei, Dai Shilin. J. Chinese Herbal Medicine, 2015 (20): 3125-3130.in Chinese.

[9]Lin Hongsheng, Li Peiwen, Wang Xiaomin. J. Chinese Journal of New Drugs, 2012, (17): 2047-2050+2055.in Chinese.

[10]Chen Bin, Jia Xiaobin. J. Chinese Herbal Medicine, 2012, (03): 592-597.in Chinese.

[11]Guo Xiaoyu, Du Jie. J. Chinese Journal of clinicians, 2015, (09): 91-93.in Chinese.

[12]Zhou Huandi, Li Li. J. Chinese Folk Medicine, 2012, (01): 43-45.in Chinese.

[13] Zheng Yuanjun, Zhang Linlin, Peng Zhiping. J. New Chinese Medicine, 2015, (01): 252-253.in Chinese.

[14] Wang Lifang, Gao Wenyuan.J. China Folk Therapy, 2016 (07): 8-10.in Chinese.

[15]Huang Yan, Zheng Xianguo. J. Chinese Modern Chinese Medicine, 2012, (07): 44-48.in Chinese. 